\title{
Building a better bridge: Remodeling, recovery, and a better understanding of the biologic foundation of mechanical circulatory support
}

\author{
William Hiesinger, MD, and Pavan Atluri, MD
}

\footnotetext{
From the Division of Cardiovascular Surgery, Department of Surgery, University of Pennsylvania School of Medicine, Philadelphia, Pa.

Disclosures: Authors have nothing to disclose with regard to commercial support.

Received for publication Aug 31, 2015; revisions received Sept 8, 2015; accepted for publication Sept 9, 2015. Address for reprints: Pavan Atluri, MD, Division of Cardiovascular Surgery, Department of Surgery, University of Pennsylvania, 6 Silverstein Pavilion, 3400 Spruce St, Philadelphia, PA 19072 (E-mail: pavan.atluri@uphs. upenn.edu).

J Thorac Cardiovasc Surg 2015;150:1342-3

$0022-5223 / \$ 36.00$

Copyright (c) 2015 by The American Association for Thoracic Surgery http://dx.doi.org/10.1016/j.jtcvs.2015.09.042
}

The global pandemic of ischemic heart disease and heart failure continues to grow despite numerous advances in both medical and surgical therapies. Myocardial calcium cycling, cell therapy, and mechanical circulatory support are all promising and diverse areas for heart failure treatment. For eg, recent advances in our understanding of calcium cycling mechanisms in cardiomyocytes have produced novel therapies for heart failure, particularly those that target sarcoplasmic reticulum calcium handling proteins. Sarcoplasmic reticulum calcium ATPase has provided a particularly fertile and promising area for modification, both by increased expression via gene therapy and through upregulation of small protein effectors such as the SUMOI and S100Al genes, the latter of which may also positively influence mitochondrial energetics. ${ }^{1}$ Similarly, cell therapy for heart failure has made great strides in the past decade, and numerous phase 1 though 3 clinical trials are enrolling or are underway in the United States and Europe utilizing a wide variety of cell types and delivery systems. However, a large chasm still exists between efficacious and optimal with regard to cell type, route, dose, and timing of delivery.

The progress and utility of mechanical circulatory support represents perhaps the most substantial recent step in the treatment of heart failure. Since the Randomized Evaluation of Mechanical Assistance for the Treatment of Congestive Heart Failure trial ${ }^{2}$ permanently unchained ventricular assistance from transplant eligibility, radical innovations to pump design and surgical technique have made destination therapy continually safer and have dramatically improved quality of life. However, the vicious cycle of myocyte death, mechanical inefficiency, adverse remodeling, and eventual heart failure is exceedingly difficult to break and the previous enthusiasm for mechanical circulatory support as a bridge to recovery has been tempered by the reality that only a small percentage of patients will recover sufficiently to allow device removal. ${ }^{3}$ It is clear that mechanical unloading of the left ventricle can offer substantial

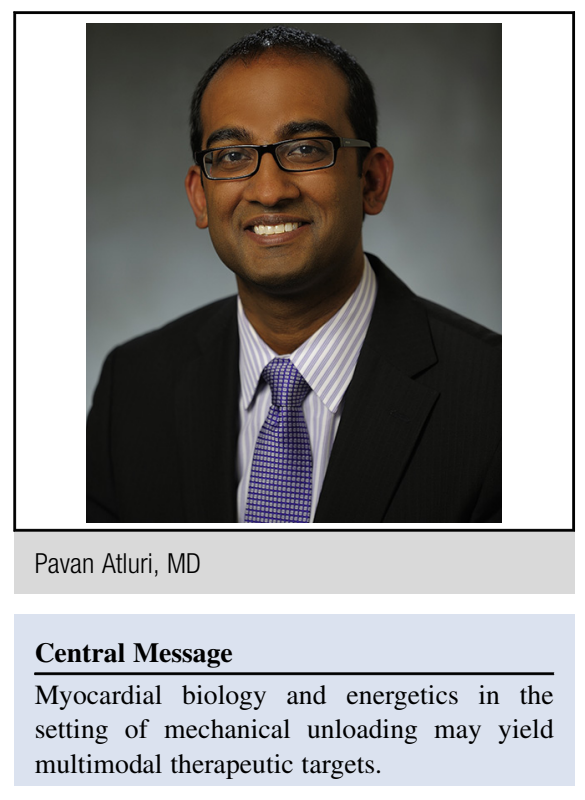

See Article page 1332.

clinical benefits, including decreased pulmonary congestion, reduced volume overload, and improved cardiac geometry; however, it is less obvious how mechanical circulatory support effects reverse remodeling at a cellular level and even less obvious what needs to be done to induce true myocardial recovery that is associated with freedom from clinical heart failure events. ${ }^{4,5}$

The excellent study by Rajagopal and colleagues ${ }^{6}$ uses an ovine model of myocardial infarction with subsequent placement of a small platform catheter-based left ventricular assist device (LVAD) to elucidate how mechanical loading affects specific cardioprotective intracellular signaling pathways. They found that LVAD support inhibited $\beta$-arrestin-mediated signaling (which enhances both cardiomyocyte function and survival) while normalizing load-induced G-protein-coupled receptor signaling in the myocardial infarction-adjacent zone. These findings are important for 2 reasons. In a narrow sense, the identification $\beta$-arrestin-mediated signaling as a potential specific therapeutic target to be manipulated in synergy with mechanical circulatory support is significant. We are at the nascent stages of understanding the molecular basis and abnormal energy metabolism of myocardial dysfunction, and have even less knowledge about how ventricular 
loading conditions affect these pathways. This study offers a concrete platform for both specific treatment and further study. Recent investigations by other groups have also begun to illustrate the microstructural, ultrastructural, metabolic, and molecular consequences of mechanical unloading $^{7}$ as well as its effect on intramyocardial calcium handling. ${ }^{5}$

More broadly, these investigators are building the foundation of what will likely be the best platform for an efficacious bridge to recovery: Multimodal therapy utilizing the titration of mechanical myocardial unloading. We are already beginning to realize the power of combined treatments and have seen safety and potential efficacy in combined stem cell and LVAD therapy ${ }^{8}$ as well as elevated rates of LVAD explantation and recovery with the addition of intense pharmacologic intervention. ${ }^{9}$ To paraphrase what Braunwald said in a recent lecture ${ }^{10}$ to achieve optimal results in the battle against heart failure a combination of approaches will be needed. Looking toward the future, it is not hard to image a hybrid approach to myocardial recovery that combines small protein modulators of myocardial calcium cycling with the surgical implantation of bioengineered stem cells constructs at the time of LVAD placement in conjunction with serial endovascular injections of a sustained release stem cell solutions.

The major limitation of the study by Rajagopal and colleagues ${ }^{6}$ is that the sample size is small and the scope of the investigation is rather narrow. This is reflective more of the amazing complexity of the biologic and mechanical interactions between the heart and assist devices and the need for further investigations of this kind than the quality of the research. The myocardial adaptations to ischemia and heart failure and then subsequent adaptations to ventricular unloading yield a unique and changing pattern of metabolic physiology and cellular stress responses. Within this intricate web may lie the building blocks that bridge reversion toward a normal myocardial phenotype and clinical recovery.

\section{References}

1. Gorski PA, Ceholski DK, Hajjar RJ. Altered myocardial calcium cycling and energetics in heart failure-a rational approach for disease treatment. Cell Metab. 2015;21:183-94

2. Rose EA, Gelijns AC, Moskowitz AJ, Heitjan DF, Stevenson LW, Dembitsky W, et al. Randomized Evaluation of Mechanical Assistance for the Treatment of Congestive Heart Failure (REMATCH) study group. Long-term use of a left ventricular assist device for end-stage heart failure. N Engl J Med. 2001;345 $1435-43$.

3. Dandel M, Knosalla C, Hetzer R. Contribution of ventricular assist devices to the recovery of failing hearts: a review and the Berlin Heart Center Experience. Eur J Heart Fail. 2014;16:248-63.

4. Mann DL, Barger PM, Burkhoff D. Myocardial recovery and the failing heart: myth, magic, or molecular target? J Am Coll Cardiol. 2012;60:2465-72.

5. Castillero E, Akashi H, Pendrak K, Yerebakan H, Najjar M, Wang C, et al. Attenuation of the unfolded protein response and endoplasmic reticulum stress after mechanical unloading in dilated cardiomyopathy. Am J Physiol Heart Circ Physiol. 2015;309:H459-70.

6. Rajagopal K, Saha P, Mohammed I, Sanchez PG, Li T, Wu ZJ, Griffith BP. Effects of small platform catheter-based left ventricular assist device support on regional myocardial signal transduction. J Thorac Cardiovasc Surg. 2015; 150:1332-41.

7. Diakos NA, Selzman CH, Sachse FB, Stehlik J, Kfoury AG, Wever-Pinzon O, et al. Myocardial atrophy and chronic mechanical unloading of the failing human heart: implications for cardiac assist device-induced myocardial recovery. J Am Coll Cardiol. 2014;64:1602-12.

8. Ascheim DD, Gelijns AC, Goldstein D, Moye LA, Smedira N, Lee S, et al. Mesenchymal precursor cells as adjunctive therapy in recipients of contemporary left ventricular assist devices. Circulation. 2014;129:2287-96.

9. Birks EJ, George RS, Hedger M, Bahrami T, Wilton P, Bowles CT, et al. Reversal of severe heart failure with a continuous-flow left ventricular assist device and pharmacological therapy: a prospective study. Circulation. 2011;123:381-90.

10. Braunwald E. The war against heart failure: the Lancet lecture. Lancet. 2015;385 812-24. 